\title{
DOI 10.18699/BGRS/SB-2020-196 \\ Exploring interaction between metabolic pathways involved in pigmentation of barley spike
}

Anastasiia Yu. Glagoleva

ICG SB RAS, Novosibirsk, Russia glagoleva@bionet.nsc.ru

Sergei R. Mursalimov

ICG SB RAS, Novosibirsk, Russia mursalimov@bionet.nsc.ru

Olesya Yu. Shoeva

ICG SB RAS, Novosibirsk, Russia olesya_ter@bionet.nsc.ru
Nikolay A. Shmakov

ICG SB RAS, Novosibirsk, Russia

shmakov@bionet.nsc.ru

Natalia V. Gracheva

VSTU, Volgograd, Russia

gracheva.tasha@yandex.ru

Elena K. Khlestkina

ICG SB RAS, Novosibirsk, Russia

VIR, Saint-Petersburg, Russia

khlest@bionet.nsc.ru
Aleksandr V. Vikhorev

ICG SB RAS, Novosibirsk, Russia

NSU, Novosibirsk, Russia

vikhorev@bionet.nsc.ru

Tatjana V. Kukoeva

ICG SB RAS, Novosibirsk, Russia

kukoeva@bionet.nsc.ru

\begin{abstract}
The color of barley grain (Hordeum vulgare L.) can be caused by synthesis and accumulation of various pigments. Besides green chlorophyll, pigmentation of barley spike can also be caused by two types of phenolic compounds: purple or blue anthocyanins and black melanins. The chemical and genetic nature of anthocyanins are well-known, while the metabolic pathways implicating in melanin formation remains less understood. The aim of the study was to explore the interaction between metabolic processes involved in melanin, anthocyanins and chlorophyll formation using the precise genetic models - near-isogenic lines (NILs) with different composition of pigments. Using marker-assisted selection we have developed two hybrid NILs: i:BwBlpalm with chlorophyll deficiency and melanin accumulation and i:BwBlpAnt1Ant2 with simultaneous melanin and anthocyanin accumulation in grain. The analysis of these lines allows us to suggest that melanin biosynthesis affects plant metabolic processes significantly. Although melanin synthesis occurs chlorophyll/photosynthesis independently, it probably has an ability to suppress the photosynthesis in barley spike. Unlike that, the presence of dominant Blp allele increases the anthocyanins accumulation in grain pericarp.
\end{abstract}

Keywords - barley, near-isogenic lines, phenylpropanoids, anthocyanins, melanin, chlorophyll

Motivation and aim

\section{Motivation}

The color of barley grain (Hordeum vulgare L.) can be caused by synthesis and accumulation of various pigments. Besides green chlorophyll, two types of phenolic compounds are involved in pigmentation of barley spike: purple or blue anthocyanins and black melanins. Different genes control the accumulation of these pigments: the complementary genes Ant1 and Ant 2 determine synthesis of purple anthocyanins in grain pericarp; the $B l x$ genes control accumulation of blue anthocyanins in aleurone layer, while melanins are formed under control of the Blp gene in glumes and pericarp tissues [1]. The biochemical pathway leading to anthocyanins is wellknown, while melanin remains the less studied pigment in plants and the metabolic pathways involved are not clear enough. The comparative RNA-seq analysis between melanin-accumulating NIL and its parental cultivar Bowman have revealed the influence of the Blp locus on expression of more than a thousand genes. Among these, genes belonging to phenylpropanoid and fatty acids biosynthesis pathways were the most represented and upregulated in black-colored NIL
[2]. Comparative microscopy analysis of the NILs grains at early, soft and hard dough developmental stages revealed the intracellular dark structures co-localized with the red autofluorescence signal of chlorophyll in cells of pericarp and hulls of the $\mathrm{i}$ :BwBlp line starting from early dough stage. Thus, the melanin pigments were showed to be formed and accumulated in chloroplasts [3]. The data served as the basis for studying, on one hand, the interaction between biosynthesis pathways of phenolic compounds - melanin and anthocyanins, which together can be accumulated in barley grain pericarp cells,- and relationships between melanin formation and chlorophyll synthesis (and photosynthesis), which together can be present in chloroplasts pericarp cells, on the other hand.

\section{Aim}

The aim of the study was to explore interaction between metabolic processes involved in melanin, anthocyanins and chlorophyll formation using precise genetic models - nearisogenic lines (NILs) with different composition of pigments.

\section{Methods}

To investigate melanin/chlorophyll and melanin/anthocyanins interactions two hybrid NILs i:BwBlpalm with chlorophyll deficiency and melanin accumulation and $\mathrm{i}: \mathrm{BwBlpAnt1Ant2}$ with simultaneous melanin and anthocyanin accumulation in grain were developed using marker-assisted selection. The initial Bowman NILs i:BwBlp (NGB20470), i:Bwalm (NGB20419), i:BwAnt1Ant2 (NGB22213) were obtained from NordGen (Alnarp, Sweden). The study of metabolic pathways interaction was performed with comparative gene expression analysis, including RNA-seq approach and RT-PCR.

\section{Results}

Using microsatellite markers linked to the Alm [4] and Blp [1] loci, the homozygote hybrid plants for these loci were selected from the $\mathrm{F}_{2}$ population generated by hybridization of $\mathrm{i}: \mathrm{Bw} B l p$ with i:Bwalm. The selected plants are characterized by partial albinism phenotype in stem node accompanying the chlorophyll insufficiency in pericarp and glume, and melanin pigmentation in grain. Inheritance of these traits in homozygote and stability of phenotype were checked in $\mathrm{F}_{3}$ families. Similar approach was used to create the hybrid line i:BwBlpAnt1Ant2 characterized by simultaneous accumulation of melanins and anthocyanins in pericarp 
tissues. In this case, the intragenic markers for the Ant1 [5] and Ant2 [6] genes were used. The developed lines represent a convenient genetic model for comparative studies of interaction between metabolic pathways underlining pigments synthesis.

In the case of melanin/chlorophyll interaction, the fact of obtaining $\mathrm{i}:$ BwBlpalm line allows us to suggest chlorophyll/photosynthesis-independent melanin synthesis. At the same time, the data of comparative transcriptomic analysis demonstrated downregulation of chloroplast genes in NIL accumulated melanin. Thus, it allows us to suggest the tendency to photosynthesis suppression in response to melanin synthesis.

In the case of melanin/anthocyanins pathways relationships, we observed the appearance of anthocyanin pigmentation at an earlier stage of spike development than the appearance of melanin pigment in $\mathrm{i}: \mathrm{BwBlpAnt1Ant2}$ line, as well as the increased content of anthocyanins in the spike in the hybrid line in compared to the parental NIL. This observation points that the same precursor compounds are involved in the biosynthesis of anthocyanins and melanin. The comparative RNA-seq analysis of $\mathrm{i}: \mathrm{BwBlpAnt1Ant2}$ and its parental NILs i:BwAnt1Ant2 and i:BwBlp at the different stages of spike development confirms upregulation of the key phenylpropanoid biosynthesis genes at the early stage in all NILs in comparison to Bowman. However, it can be concluded that the presence of Blp locus significantly affects genes expression in barley spike and activates unique genes of melanogenesis.

\section{Acknowledgment}

Comparative transcriptomic and cytological analysis of barley NILs were supported the Russian Science Foundation grant No.16-14-00086, development and analysis of the i:BwBlpalm line were supported by the Russian Science Foundation grant No.19-76-00018, development and analysis of the i:BwBlpAnt1Ant2 line were supported by the RFBR grant No. 20-316-80016.

\section{References}

[1] Шоева О.Ю. и др. (2018). Гены, контролируюшие синтез флавоноидных и меланиновых пигментов ячменя. Вавиловский журнали генетики и селекции. 22(3):333-342.

[2] Glagoleva A.Yu. et al. (2017). Metabolic pathways and genes identified by RNA-seq analysis of barley near-isogenic lines differing by allelic state of the Black lemma and pericarp (Blp) gene. BMC Plant Biology. 17(Suppl 1):182

[3] Shoeva O.Yu. et al. (2020). Melanin formation in barley grain occurs within plastids of pericarp and husk cells. Scientific Reports. 10:179.

[4] Shmakov N.A. et al. (2016). Identification of nuclear genes controlling chlorophyll synthesis in barley by RNA-seq. BMC Plant Biology. 16: 245.

[5] Himi E., Taketa S. (2015). Isolation of candidate genes for the barley Ant1 and wheat Rc genes controlling anthocyanin pigmentation in different vegetative tissues. Molecular Genetics and Genomics. 290(4):1287-1298.

[6] Cockram J. et al, (2010). Genome-wide association mapping to candidate polymorphism resolution in the unsequenced barley genome. Proceedings of the National Academy of Sciences USA. 107(50):21611-21616 